\title{
REALIZABILITY OF REPRESENTATIONS IN CYCLOTOMIC FIELDS
}

\author{
BURTON FEIN ${ }^{1}$
}

\begin{abstract}
In this paper sufficient conditions are given for a real valued complex irreducible character $\chi$ to be written in $Q(\chi)\left(\varepsilon_{q}\right), \varepsilon_{q}$ a primitive $q$ th root of unity, $q$ a prime dividing the order of the group.
\end{abstract}

Let $T$ be an irreducible complex representation of the finite group $G$ and suppose that the character $\chi$ of $T$ is real valued. In [3, Theorem 3, p. 712], R. Brauer proved that $T$ can be written in $Q(\chi)\left(\varepsilon_{n}\right)$, the field generated over $Q(\chi)$ ( $Q$ the rationals) by a primitive $n$th root of unity $\varepsilon_{n}$, where $n$ divides the order $|G|$ of $G$ and is either an odd prime or a power of 2 . In this note we give a sufficient condition for $T$ to be written in $Q(\chi)\left(\varepsilon_{q}\right)$ with $q$ a prime divisor of $|G|$.

THEOREM. Let $T$ be an irreducible complex representation of the finite group $G$ and suppose that the character $\chi$ of $T$ is real valued. Assume there is a prime divisor $q$ of $|G|$ and an element $\alpha \in G$ of order $q$ such that $T(\alpha)$ does not have 1 as an eigenvalue, $2(q-1) \nmid \chi(1)$, and $Q(\chi) \cap Q\left(\varepsilon_{q}\right)=Q$. Then $T$ can be written in $Q(\chi)\left(\varepsilon_{q}\right)$.

Proof. By the Brauer-Speiser theorem [3, p. 711] the Schur index of $T$ over $Q$ is 1 or 2 . Since the result is clear if the Schur index is 1 , we may assume that $T$ has Schur index 2 . In particular, $\chi(1)$ is even so $q$ is odd. Let $K=Q(\chi)$ and let $S$ be the $K$-representation of $G$ such that $S \sim 2 T$. Since $T(\alpha)$ does not have 1 as an eigenvalue, neither does $S(\alpha)$. Let $m=2 \chi(1)$ and set $A=S(\alpha)$. Denote by $K[A]$ the subring of the $m \times m$ matrices over $K, M_{m}(K)$, generated by $A$ and the $m \times m$ scalar matrices. $K[A]$, being a homomorphic image of the group algebra over $K$ of the cyclic group of order $q$, is a commutative semisimple ring. Hence $K[A]$

Received by the editors July $17,1972$.

AMS (MOS) subject classifications (1970). Primary $20 \mathrm{C} 15$.

Key words and phrases. Schur index, Brauer-Speiser theorem, rational division algebra, Hasse invariant.

1 This work was done under the sponsorship of NSF Grant GP-29068.

(C) American Mathematical Society 1973 
is a field (isomorphic to $K\left(\varepsilon_{q}\right)$ ) if and only if it has no zero divisors. Let $f(x)=x^{q-1}+x^{q-2}+\cdots+x+1$ and suppose $P$ is an invertible $m \times m$ complex matrix with $P^{-1} A P$ diagonal. Since $A^{q}=I$ and 1 is not an eigenvalue, $f\left(P^{-1} A P\right)=P^{-1} f(A) P=0$. Since $K \cap Q\left(\varepsilon_{q}\right)=Q, f(x)$ is irreducible in $K[x]$ and so $f(x)$ is the minimal polynomial of $A$. If $g(x)$ and $h(x)$ are in $K[x]$ with $g(A) h(A)=0$, then $f(x)$ divides $g(x) h(x)$ and, by the irreducibility of $f(x), f(x)$ divides $g(x)$ or $h(x)$. Thus $g(A)$ or $h(A)$ equals 0 and so $K[A] \cong K\left(\varepsilon_{q}\right)$. Now $K[A]$ is a subalgebra of the enveloping algebra of $S$. By the theory of the Schur index (see $[4, \S 70]$ ) this enveloping algebra is isomorphic to $M_{n}(D)$ where $D$ is a division algebra central over $K$, $[D: K]=4$ and $n=\chi(1) / 2$. Thus $M_{n}(D) \supset K\left(\varepsilon_{q}\right)$. $T$ can be written in $K\left(\varepsilon_{q}\right)$ if and only if $D \otimes_{K} K\left(\varepsilon_{r}\right) \sim K\left(\varepsilon_{q}\right)$. If this was not the case, then $D \otimes_{K} K\left(\varepsilon_{q}\right)$ would have index 2 and $M_{q-1}(D)$ would be the algebra of least degree similar to $D$ and containing a subfield isomorphic to $K\left(\varepsilon_{q}\right)$ [1, Theorem 23, p. 60]. In this case we would also have $q-1 \mid n$ and so $2(q-1) \mid \chi(1)$, a contradiction. Thus $K\left(\varepsilon_{q}\right)$ splits $D$, proving the theorem.

REMARKS. (1) If $T(\alpha)$ does not have 1 as an eigenvalue then $(q-1) \mid \chi(1)$. For, since $\left[K\left(\varepsilon_{q}\right): K\right]=q-1$ and $\chi(\alpha) \in K$, every distinct eigenvalue of $T(\alpha)$ occurs with equal multiplicity and each $\varepsilon_{q}^{i}, 1 \leqq i \leqq q-1$, occurs. We also note that if $\chi(1)=d(q-1)$ and $Q(\chi) \cap Q\left(\varepsilon_{q}\right)=Q$, then $T(\alpha)$ does not have 1 as an eigenvalue if and only if $\chi(\alpha)=-d$.

(2) Let $G$ be generated by $x, y$, and $c$ subject to the relations $x^{7}=1$, $y^{-1} x y=x^{3}, y^{6}=c, c^{2}=1$, and $c$ central. By results of [2] or [5] $G$ has an irreducible complex representation $T$ of Schur index 2 having a rational character $\chi$ of degree 6 . Let $S$ be the rational representation with $S \sim 2 T$. The enveloping algebra of $S$ is $M_{3}\left(D_{7, \infty}\right)$ where $D_{7, \infty}$ denotes the rational division algebra with invariant $\frac{1}{2}$ at 7 and $\infty$ and invariant 0 at all other primes of $Q$. Let $q=3$ and $\alpha=y^{4}$. Then $2(q-1) \nmid \chi(1)$ and $Q(\chi) \cap Q\left(\varepsilon_{q}\right)=Q$. Since 7 splits in $Q\left(\varepsilon_{3}\right), Q\left(\varepsilon_{3}\right)$ does not split $D_{7, \infty}$ [1, Chapter 9]. Thus $T$ cannot be written in $Q\left(\varepsilon_{3}\right)$. This shows that the hypothesis that 1 is not an eigenvalue cannot be dropped even if we assume $q-1 \mid \chi(1)$. (In this case $\chi\left(y^{4}\right)=0$ so 1 is clearly an eigenvalue.)

(3) $S_{4}$ has a faithful absolutely irreducible rational representation $T$ of degree 3. The enveloping algebra of this representation is $M_{3}(Q)$. Since $\left[Q\left(\varepsilon_{3}\right): Q\right] \nmid 3, M_{3}(Q)$ does not have a subalgebra isomorphic to $Q\left(\varepsilon_{3}\right)$ even though $T((123))^{3}=1$. The problem is that $T(123)$ has 1 as an eigenvalue and so $Q[T((123))]$ is not a field. More generally, Charles Ballantine has observed that if $A \in M_{n}(K)$, then $K[A]$ is a field if and only if its minimal polynomial is irreducible in $K[x]$. If $A \in M_{n}(Q)$ and $A^{q}=I, q$ a prime, then $(A-1)\left(A^{q-1}+A^{q-2}+\cdots+A+1\right)=0$. If $A \neq I$ and 1 is an eigenvalue of $A$, then neither factor equals 0 so $Q[A]$ contains zero divisors. 


\section{REFERENCES}

1. A. A. Albert, Structure of algebras, Amer. Math. Soc. Colloq. Publ., vol. 24, Amer. Math. Soc., Providence, R.I., 1939. MR 1, 99.

2. M. Benard, Quaternion constituents of group algebras, Proc. Amer. Math. Soc. 30 (1971), 217-219.

3. R. Brauer, Applications of induced characters, Amer. J. Math. 69 (1947), 709-716. MR 9, 268.

4. C. W. Curtis and I. Reiner, Representation theory of finite groups and associative algebras, Pure and Appl. Math., vol. 11, Interscience, New York, 1962. MR 26 \#2519.

5. K. L. Fields, On the Brauer-Speiser theorem, Bull. Amer. Math. Soc. 77 (1971), 223. MR 42 \#3192.

Department of Mathematics, Oregon State University, Corvallis, Oregon 97330 